\title{
Gradhiva
}

GRADHIV

Revue d'anthropologie et d'histoire des arts

$32 \mid 2021$

Livres sorciers

\section{Le dernier livre}

The Last Book

\section{Pierre Déléage}

\section{(2) OpenEdition}

Journals

Édition électronique

URL : https://journals.openedition.org/gradhiva/5606

DOI : 10.4000/gradhiva.5606

ISSN : 1760-849X

\section{Éditeur}

Musée du quai Branly Jacques Chirac

\section{Édition imprimée}

Date de publication : 24 mars 2021

Pagination : 144-156

ISBN : 978-2-35744-132-3

ISSN : 0764-8928

\section{Référence électronique}

Pierre Déléage, « Le dernier livre », Gradhiva [En ligne], 32 | 2021, mis en ligne le 02 avril 2021, consulté le 25 mai 2021. URL : http://journals.openedition.org/gradhiva/5606 ; DOl : https://doi.org/10.4000/ gradhiva.5606

(c) musée du quai Branly 
$5 \times x-3=6+5$

$950 x+3 \times$ $+5$ 8. $3 x+3 x+2=$ $4 x^{2}+3 x$ $3 x^{2}-2+2$ $y^{2}=x^{2}+\frac{7}{2}$ $2 \times 3.62=$ $x^{2}+2>0$ $x^{2}+20202$ $x+1$ 8 Sit .
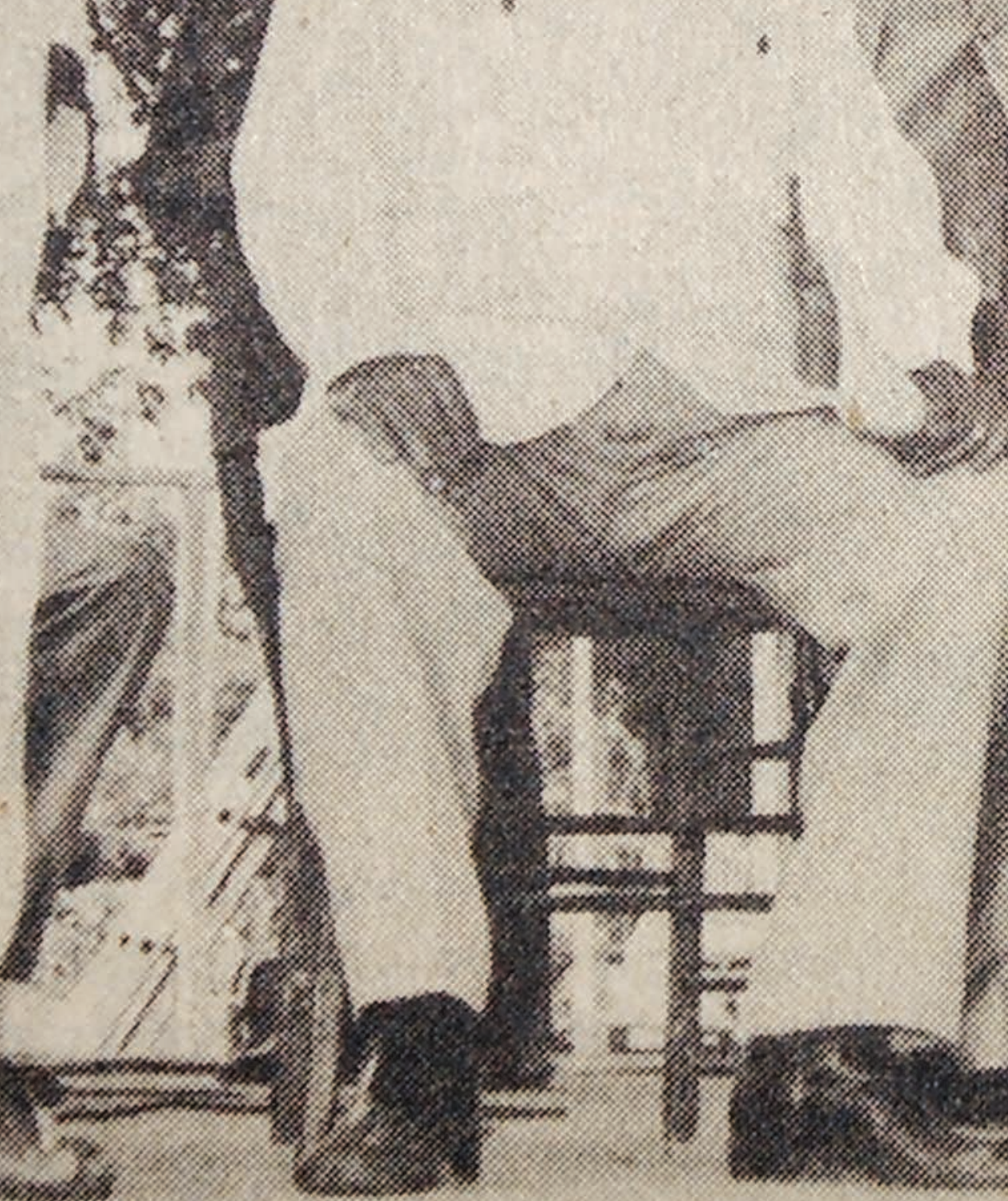
sos
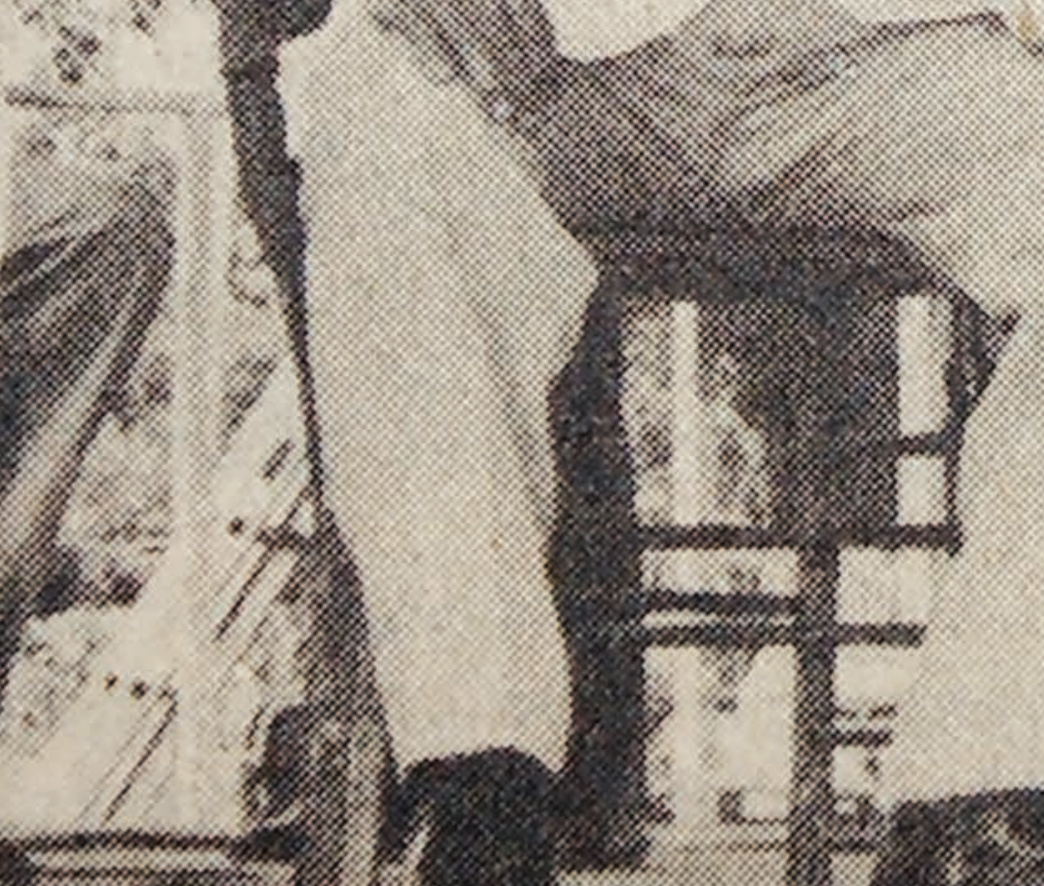

)
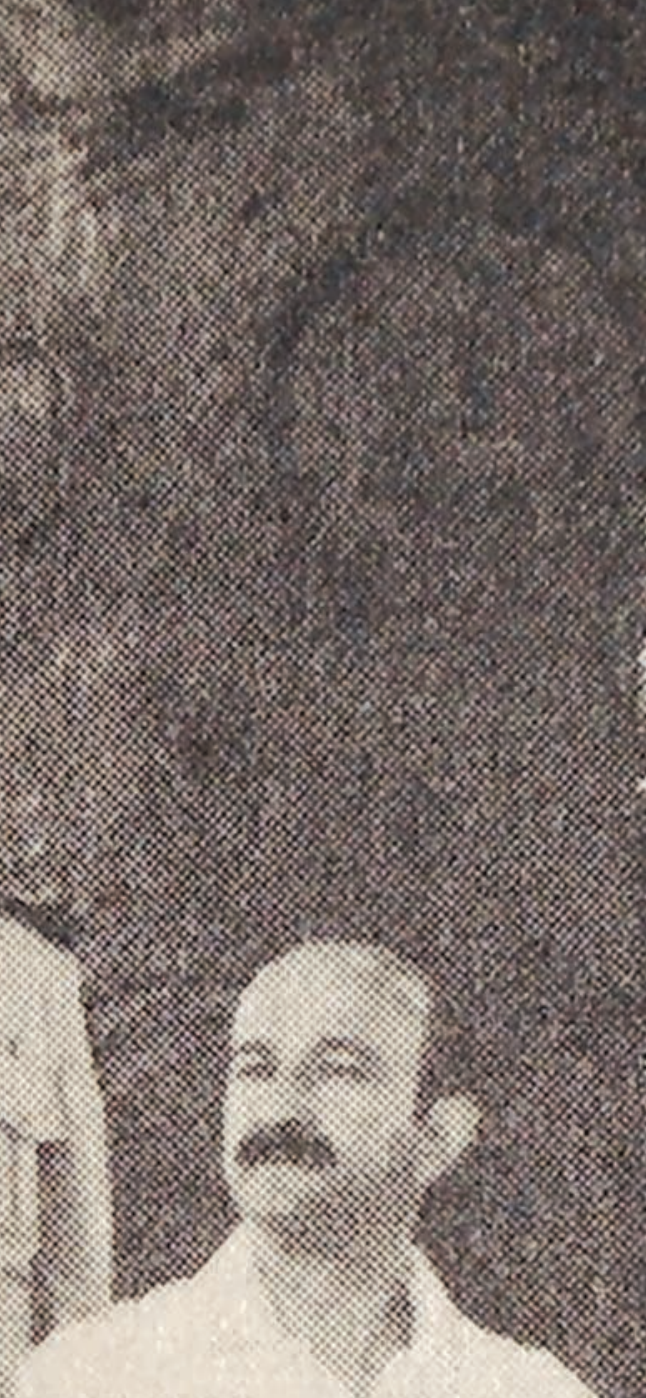

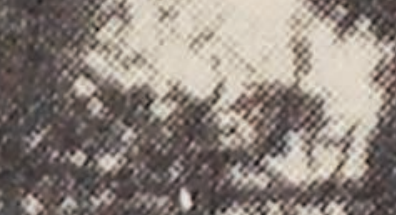
2 Muth tr. . 5.<smiles>[AlH2]</smiles><smiles>c1ccccc1</smiles>

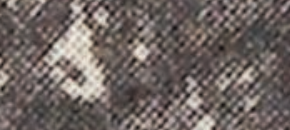
. 
Le dernier livre

\section{Pierre Déléage}


Au nord de la péninsule du Yucatán, les Mayas du village de Xocén se transmettent un récit à propos d'un livre aux propriétés stupéfiantes. II s'agit non seulement d'un livre vivant, sans auteur humain, apparu lors de la création du monde, mais aussi d'un livre qui contient la totalité du savoir, la chronique exhaustive du passé comme les prophéties de l'avenir. Les Mayas de Xocén disent que ce livre leur a été donné par Dieu. Ils disent aussi qu'il leur a été dérobé et qu'il est désormais conservé quelque part aux États-Unis. Ils expliquent ainsi la spoliation coloniale et la permanence de la domination impérialiste. Ils prédisent enfin qu'un jour le livre leur reviendra.

1. «Solicitan la búsqueda del Libro de profecias al Presidente de la República», 25 octobre 1990, in Terán Contreras et Rasmussen (éd.) 2007:189 et 194.

2. «Xocén recibe el Presidente con peticiones y quejas», Diario de Yucatán, 26 octobre 1990; voir aussi «Campesinos de Xocén solicitan créditos para impulsar actividades agropecuarias», Novedades de Yucatán, 26 octobre 1990. Toutes les traductions sont de l'auteur.
Le 26 octobre 1990, deux ans après le début de son mandat, le président du Mexique Carlos Salinas de Gortari, investi dans la campaǵne des élections législatives, se rendit brièvement dans un petit village maya qui ne comptait alors guère plus d'un millier d'habitants, Xocén.

Son hélicoptère atterrit sur un terrain défriché pour l'occasion et un autobus qui arborait fièrement le nom d'Emiliano Zapata le conduisit un kilomètre plus loin, jusqu'à la place centrale du village. Il était 11 h 20 lorsque la garde présidentielle le fit monter sur une estrade où il s'assit, à l'ombre d'un arbre gigantesque, entre le gouverneur de l'État du Yucatán et le maire de la ville voisine, Valladolid. Face à eux, une foule de Mayas en grande partie monolingue, attirée par les festivités prévues dans la journée.

Au signal d'un militaire, le représentant du conseil des anciens de Xocén, Concepción Canul Nahuat, remit cérémoniellement au président, en les accompaǵnant de quelques mots en maya, une liasse de pétitions dans lesquelles étaient sollicités, entre autres choses, le pavage des huit kilomètres de la route menant au village, la fermeture de trois débits de boissons clandestins et la construction d'un puits, d'un réseau électrique additionnel, d'une nouvelle église et d'une bibliothèque. Le président de la République, concentré, prit des notes dans un petit carnet.

Le chef du conseil suprême maya, Mario Monforte Garrido, qui s'était déplacé pour l'occasion, préféra quant à lui insister sur l'importance culturelle et religieuse du village. Il le qualifia même de «centre du monde». Puis il invita le président à fournir aux villageois l'aide nécessaire pour qu'ils puissent enfin retrouver leur livre sacré. Dans la pétition à l'origine de cette demande, maintenant entre les mains du président, il était en effet question d'un livre «qui avait été volé au début du XIX ${ }^{\mathrm{e}}$ siècle». Il ne s'aǵissait certes là que de «l'une des innombrables souffrances causées par la domination espaǵnole $\mathbf{1}$ ». Mais la République était tout de même sommée de mettre un terme à cette injustice héritée de l'époque coloniale.
Le président Salinas de Gortari, calvitie avancée et moustache fournie, prit alors le micro. Il fit un de ces discours lénifiants que les hommes politiques ont l'habitude de prononcer lors des campagnes électorales. Il promit beaucoup. Il demanda aussi aux habitants de Xocén de dresser une liste de leurs requêtes classées cette fois par ordre de priorité. Puis il conclut sa brève harangue par ces mots :

J'ai pris acte de la demande de récupération du livre sacré et je vais sur-lechamp donner des instructions en ce sens au directeur de l'Institut national indigéniste ${ }^{2}$.

Il était déjà 12 h 10, le président devait partir. Sa tournée du Yucatán n'était pas encore achevée et d'autres villages l'attendaient. Il était resté moins d'une heure. Ce fut la première et la dernière fois qu'un président de la République foula le sol du village maya de Xocén.

$*$

Pourquoi le président du Mexique avait-il pris la peine de faire un détour par ce petit village? L'année précédente un groupe de cinq enseiǵnants issus des milieux urbains du Yucatán et de l'État limitrophe de Tabasco était venu à Xocén pour fonder, sous la direction de María Alicia Martínez Medrano, un Laboratoire de théâtre paysan et indigène. Ils avaient obtenu l'accord des villageoois puis, grâce au carnet d'adresses bien rempli de leur directrice, le soutien de l'Institut national indigéniste (INI) et du conseil suprême maya (deux organisations très proches du pouvoir en place, c'està-dire du Parti révolutionnaire institutionnel [PRI], le parti du président, hégémonique au Mexique depuis la Seconde Guerre mondiale). L'une des missions de ces institutions était d'encourager les «activités culturelles dans les zones indigènes »; le groupe de théâtre avant-gardiste était donc totalement en phase avec le paysage politique local (Navarrete Maya 2000; Muñoz Castillo 2001: 73-80). 
Le dernier livre

Pierre Déléage



Écriteau à l'entrée du Laboratoire théâtral de Xocén. Photographie de Pierre Déléage, 2012. 


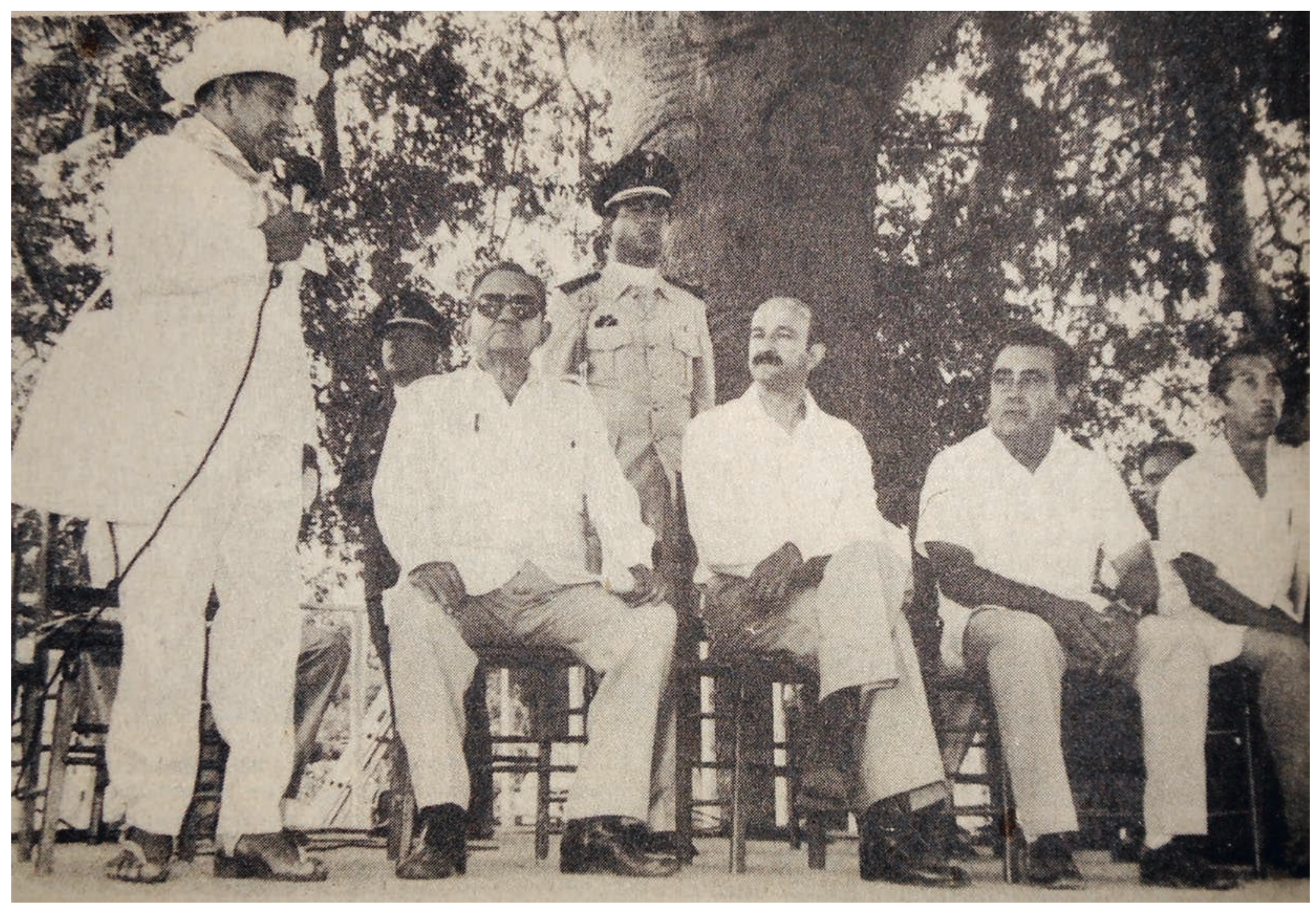

Le Président mexicain Carlos Salinas de Gortari lors de sa visite à Xocén. De gauche à droite au premier plan, Mario Monforte Garrido (chef du conseil suprême maya), Victor Manzanilla Shaffer (gouverneur du Yucatán), Carlos Salinas de Gortari (Président de la République), William Centeno Machain (maire de Valladolid) et Secundino Pech Couoh (commissaire municipal de Xocén). Photographie prise à Xocén par Isidro Avlia Villacis. Diario de Yucatán, 26 octobre 1990. Droits réservés. 
Il s'installa à demeure et proposa rapidement un riche programme d'activités: tous les soirs, les habitants de Xocén, jeunes et adultes, pouvaient venir apprendre des danses folkloriques, mettre en pratique les principes de la biomécanique de Meyerhold, répéter des fraǵments de pièces de théâtre et se défouler dans des exercices d'improvisation. Ce fut un immense succès. Plus d'une centaine de villageois participèrent régulièrement, enthousiastes, au Laboratoire indigéniste. Un grand espace scénique en plein air fut accommodé au cour du village. Et bientôt une pièce de théâtre originale fut créée.

Intitulée Xocén, prends-moi en compte, j'existe, ne m'oublie pas, elle mettait en scène de grands mouvements chorégraphiques et des reproductions de cérémonies plus ou moins traditionnelles. Le groupe d'enseignants, à la recherche d'une certaine essence maya, déroulait une sévère critique de la civilisation moderne, occidentale et impérialiste, et puisait son inspiration dans le Popol Vuh, un livre manuscrit rédigé en quiché à l'époque coloniale, témoin des traditions orales des lointains Mayas des hautes terres du Guatemala. Les Mayas de Xocén étaient entièrement étrangers à ces traditions mais les enseignants souhaitaient ardemment les voir exalter et s'approprier ce passé mythifié. En somme tout cela était plutôt amusant.

En avril 1990, la pièce fut représentée à México, la capitale du pays, dans la troisième section du parc de Chapultepec. C'est alors que la troupe sollicita la visite du président. On pourrait même croire que le titre de la pièce lui était directement adressé 3 .

$*$

Trois semaines après le discours présidentiel de Xocén, le conseil du village s'accorda sur une liste de dix-huit requêtes hiérarchisées par ordre d'importance. L'accès à l'eau potable, le pavage de la route, la construction d'une bibliothèque et d'une école secondaire ou encore le financement de plusieurs coopératives arrivaient en tête de la liste. Ce n'est qu'en dix-huitième et dernière position que l'on retrouvait la «sollicitation d'une recherche du livre sacré de Xocén ${ }^{4} »$. Cela suffit toutefois pour qu'Arturo Warman, autrefois enfant terrible de l'anthropologie mexicaine, dorénavant directeur de l'INI et ami proche du président de la République, fût contraint d'effectuer le déplacement ${ }^{5}$. Le 18 novembre, dans la salle de réception du commissariat de Xocén, il recueillit de la bouche de trois villageois, au premier rang desquels Fulgencio Noh Dzib, le scribe de la communauté, les informations disponibles sur ce «livre sacré des prophéties $\boldsymbol{\sigma}$. Elles étaient édifiantes.

On peut reconstituer ce qu'entendit l'anthropologue ce jour-là à partir de documents publiés peu après. Selon Fulgencio Noh, le livre, "très épais », mesurait «un mètre sur un mètre» et il était «écrit en maya» (Terán Contreras et Rasmussen [dir.] 1992). Quand avait-il été dérobé? Pour certains, à la fin du XIXe siècle, «il y a cent vingt ans ${ }^{\text {» }}$, c'est-à-dire sous la République et non durant le régime colonial espaǵnol; pour d'autres,

cela datait même du milieu du $\mathrm{xx}^{\mathrm{e}}$ siècle, alors que le PRI était déjà aux commandes du pays. Certes, dans la pétition originelle, il était écrit que le vol avait eu lieu avant l'avènement de la République, mais c'était peut-être au prix de cette légère imprécision qu'il avait été possible de retenir l'attention de l'actuel président de cette même République. Sur la suite de l'histoire, les villageois s'accordaient davantage (Mujica 1997 : 11; López Méndez 2003 : 10; Terán Contreras et Rasmussen 2005 : 89; Licausi Pérez 2010 : 139-140; Everton 2012: 198).

\section{Le livre fut prêté une fois à Valladolid et il fut rendu. Puis il fut prêté à Chichimilá et il fut encore rendu. Puis il fut à nouveau demandé par les gens de Valladolid qui, eux, ne le retournèrent pas. On dit que de Valladolid il fut emporté à México et que là-bas il fut montré à des Américains (gringos) qui le prirent avec eux. \\ (Terán Contreras et Rasmussen [dir.] 1992: 122)}

Certains ajoutaient, entre Valladolid et México, la ville de Mérida (López Méndez 2003 : 10; Licausi Pérez 2010 : 139-140). Cette histoire des emprunts puis des vols successifs du livre des prophéties dessinait une carte très précise de la hiérarchie administrative, territoriale et démographique dans laquelle est inséré le village de Xocén. Chichimilá est la capitale du canton (cabecera municipal), Valladolid celle du district municipal (municipio), Mérida celle de l'État du Yucatán et finalement México celle du Mexique. C'était toute une échelle de domination concentrique que retraçait minutieusement le récit. On ne s'étonnera done pas que le livre ait fini entre les mains de représentants des États-Unis.

On peut imagiiner la perplexité, à l'écoute de ce récit, d'Arturo Warman, haut fonctionnaire et néanmoins anthropologue. Le livre sacré de Xocén existait-il vraiment? S'aǵissait-il d'un ancien manuscrit, volé ou perdu, à propos duquel les Mayas avaient élaboré un discours politique dénonçant l'enchâssement bureaucratique de rapports de domination auxquels ils étaient désormais soumis? Ou n'était-ce qu'une légende, une critique géopolitique que les Mayas de Xocén, voisins du complexe touristique de Cancún alors en plein développement ${ }^{9}$, avaient sardoniquement adressée au cœur du pouvoir?

La suite de l'histoire allait dans le sens de cette dernière interprétation: le livre avait donc été dérobé, en dernière instance, par les gringos des États-Unis.

\section{C'est pourquoi ce sont les Américains qui ont inventé les avions, les voies fer- rées, les satellites, les fusées, les montres, les ordinateurs et toute la technologie moderne. Tout était écrit dans le livre. \\ (Terán Contreras et Rasmussen [dir.] 1992: 122)}

Le grand livre dont les Mayas de Xocén avaient été dépossédés contenait ainsi tous les savoirs nécessaires à la domination technologique. Le récit condensait en quelques phrases la destruction d'une culture,
3. «La voz de la selva en Chapultetpec», Tiempo libre 17-23 mai 1990. À partir de 1993 l'œuvre fut intitulée Moments sacrés mayas. Sur le Laboratoire de théâtre paysan et indigène, voir: entretien de l'auteur avec Natalio Noh, Xocén, 26 novembre 2011; Dzib May 1999: 29; Terán Contreras et Rasmussen 2005: 88-91. ( 12 avril 1990, in Terán Contreras et Rasmussen (éd.) 2007: 186-188; brochure du spectacle Momentos sagrados mayas, 2012; «Laureado el Teatro Indígena de Xocén», Por Esto!, 5 janvier 2014.

4. «Relación de proyectos solicitados al Presidente de la República», 15 novembre 1990, Terán Contreras et Rasmussen (éd.) 2007: 195-197.

5. Sur Arturo Warman, voir: Gallart et Rojas Rabiela 2004; Warman etal. 1970.

6. Entretien de l'auteur avec Natalio Noh, Xocén 26 novembre 2011.

7. «Relación de proyectos solicitados al Presidente de la República», 15 novembre 1990 in Terán Contreras et Rasmussen (éd.) 2007: 195-197.

8. C'est le cas aussi de Fermín Dzib May, lors d'un entretien avec l'auteur, Xocén, 9 décembre 2011.

9. Castellanos 2010 
10. «Proponen integrar una comisión para la búsqueda del Libro sagrado de Xocén », Diario de Yucatán, 4 novembre 1990; «Carta al director del

INI del comité de búsqueda

del Libro Sagrado de Xocén»,

12 janvier 1991, in Terán

Contreras et Rasmussen (éd.)

2007: 206-207.

11. «Carta de quejas al

director del Instituto Naciona

Indigenista», 26 avril 1991,

in ibid. : 217-221.

12. «Quejas contra el directo del INI en carta al Presidente

de la República», 20 mai 1991

in ibid.: 225-228.

13. Communication

personnelle d'Agueda Ruiz

Padilla, 6 août 2012; voir aussi:

«Informe de las actividades de

búsqueda del Libro Sagrado»,

26 mars 1992, in Terán Contreras

et Rasmussen (éd.) 2007;

236-238

14. «Se inicia la búsqueda de un libro sagrado maya»,

Diario de Yucatán, 13 avril 1992;

«Recomandaciones del INAH

sobre un libro de los mayas»,

ibid., 14 avril 1992.

15. «Informe de las actividades de búsqueda del Libro Sagrado»», 26 mars 1992, in Terán Contreras et Rasmussen (éd.) 2007:

238-242; entretien de l'auteur

avec Natalio Noh, Xocén,

26 novembre 2011.

16. Mujica 1997: 10-11; Dzib

May 1999: 29; Terán Contreras

et Rasmussen 2005: 90;

Boccara 2017: 318. la perte d'une autonomie sociale, le rapport contemporain consubstantiel entre technologie, domination économique et contrôle géopolitique. Arturo Warman devait commencer à comprendre qu'il n'était peut-être plus vraiment question de retrouver un livre volé, mais de reconnaître et de réparer, si la chose était encore possible, le scandale d'une longue et lente spoliation, stratifiée et internationale, cognitive aussi bien que politique.

Puis Fulgencio Noh, ou peut-être l'un des anciens qui l'entouraient, acheva de convaincre Arturo Warman en révélant la vraie nature du livre, son ontologie spécifique.

\section{C'est un livre naturel car il ne fut fabriqué} par personne. Le livre, seul, tourne ses pages. Chaque jour une page se déploie et si quelqu'un essaie de la tourner, le livre saigne car il est vivant. (ibid.)

Le directeur de l'INI ne pouvait plus douter que la quête du livre sacré de Xocén demeurerait vaine. Un livre vivant ne se retrouve pas dans une bibliothèque, fût-elle nord-américaine. Il fallait cependant tenir la promesse du président. Balayant d'un revers de main le scepticisme que les archéologues et les anthropologues de la région étalaient dans la presse locale quant à l'existence du livre ${ }^{10}$, Arturo Warman avertit les Mayas réunis dans le commissariat de Xocén de la difficulté d'une entreprise qui impliquerait nécessairement de longues recherches et des expéditions internationales. Il évoqua également l'issue incertaine d'une telle quête. Il s'engagea néanmoins à appuyer les villageois dans leurs démarches. Puis il monta dans sa voiture de fonction et repartit séance tenante pour la capitale. Mal à l'aise, espérons-le.

\section{*}

Cinq mois après la visite d'Arturo Warman, les Mayas de Xocén constatèrent amèrement qu'aucune promesse n'avait été tenue. Ils avaient formé de leur propre initiative un «comité pour la recherche du livre sacré de Xocén», dont le secrétaire général était Fulǵencio Noh. Mais l'appui tant attendu de l'INI tardait à se concrétiser. Ils envoyèrent à Arturo Warman une complainte, puis une autre, qui toutes deux restèrent lettre morte 11. Quelques semaines passèrent. Ils décidèrent alors de s'adresser directement au président Salinas de Gortari : son subordonné devait être rappelé à l'ordre et les promesses devaient être respectées ${ }^{12}$. La démarche s'avéra efficace: le comité finit par être en mesure d'embaucher, à partir du mois d'octobre 1991, une coordinatrice chargée de faire avancer la recherche.

Aǵueda Edith Ruiz Padilla était journaliste à Uruapan del Progreso, dans le lointain Michoacán. Elle était aussi une amie de longue date de la directrice du Laboratoire théâtral de Xocén, María Alicia Martínez Medrano, et lui avait rendu d'importants services dans le passé. Aucune compétence particulière ne semble l'avoir prédestinée à sa nouvelle fonction, si ce n'est un intérêt diffus pour l'écriture ǵlyphique des sociétés mayas préhispaniques. Elle était venue à Xocén, sur l'invitation de son amie, pour apprendre la lanǵue maya et tenter de déchiffrer l'antique écriture (ne se doutant apparemment pas que les grands principes de ce déchiffrement avaient été définitivement mis en place lors de la décennie précédente par la communauté épigraphiste internationale). En somme elle s'était trouvée au bon endroit au bon moment ${ }^{\mathbf{1 3}}$.

Aǵueda Ruiz était de toute évidence imperméable aux sarcasmes des anthropologues de la région ${ }^{\mathbf{1 4}}$. Si l'on se fie aux apparences, c'est de toute bonne foi qu'elle entreprit la quête du livre de Xocén. Arturo Warman ne répondait désormais ni aux courriers ni au téléphone. Elle ne perdit cependant pas courage et continua inlassablement à essayer de faire aboutir des procédures administratives de plus en plus délicates au fur et à mesure que le temps passait. Les membres du comité de recherche s'entretinrent à propos du livre avec plus de soixante personnes, enregistrant sur cassettes des propos aussi semblables que peu concluants. La bibliothèque de Xocén s'enrichit de très nombreux ouvrages, originaux ou photocopiés, mais le livre des prophéties demeurait introuvable ${ }^{15}$

En désespoir de cause, Agueda Ruiz eut l'idée incongrue de créer un «nouveau livre sacré». Pour ce faire elle demanda aux membres du comité de recopier certains passages de manuscrits coloniaux écrits dans la langue maya du Yucatán, qu'il fallut ensuite transposer en maya moderne. Elle considérait ces extraits comme «les plus traditionnels», c'est-à-dire «reflétant une essence maya». À ces textes elle adjoignit quelques récits traditionnels issus des entretiens enregistrés par le comité de recherche ${ }^{16}$. L'ensemble du corpus fut finalement rédigé à la main par plusieurs membres du comité dans un grand livre relié en cuir rouge. Les pages furent numérotées au moyen du système numérique des Mayas de l'époque préhispanique. Un point final fut mis à ce manuscrit de plus de deux cents pages le 28 novembre 1993.

$*$

Il était temps. Le président Carlos Salinas de Gortari achevait son sexennat et le comité devait lui présenter les résultats de ses recherches lors d'une visite à Mérida, capitale du Yucatán. Le 17 mai 1994, dans les installations du secrétariat du développement rural, devant une large assemblée de paysans et de journalistes, le président débita un discours convenu sur la renaissance de l'aǵriculture au Yucatán, délivrant au passaǵe quelques chèques symboliques à des Mayas dûment sélectionnés. Pour conclure, il fit référence au livre que venait de lui remettre en mains propres Agueda Ruiz et Fulgencio Noh.

Lorsque j'ai rendu visite, il y a quelques années, au village de Xocén, on m'exposa le problème du livre perdu. Il y avait plus de cent ans qu'il avait été égaré. Pendant un siècle et demi, il avait été 


\section{Le dernier livre}

Pierre Déléage

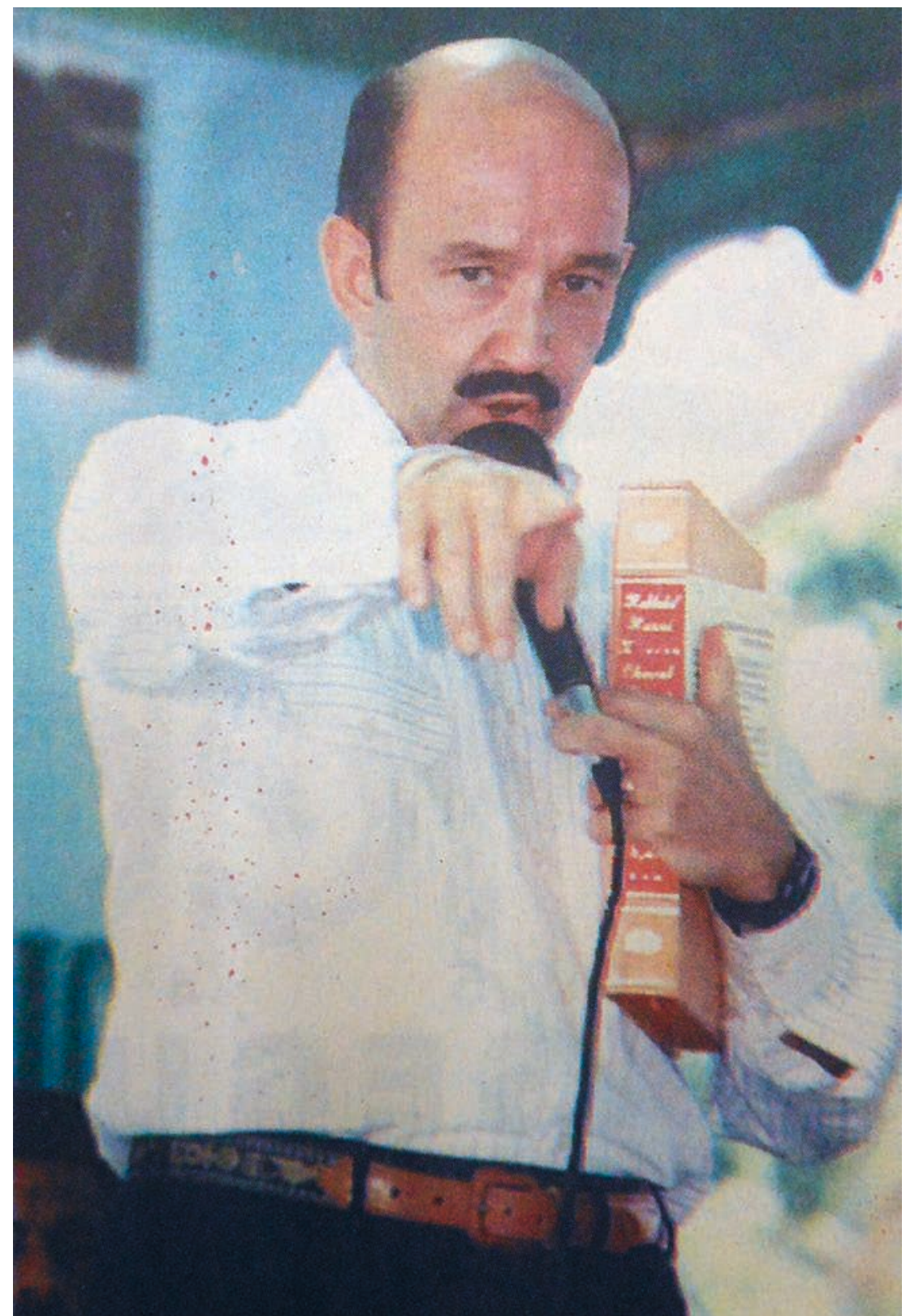

Carlos Salinas de Gortari tenant un exemplaire du «nouveau livre sacré» de Xocén. Phołographie prise à Mérida par Arturo Flota. Novedades, 17 mai 1994. 


\section{Haklahil Huuni X'ocen Chincab Dzonot}

Lakin ti Yucalpeten 


\section{Le dernier livre}

Pierre Déléage

perdu. Avec respect, nous avons proposé notre aide à la communauté pour qu'elle puisse retrouver sa mémoire, celle de ses traditions, celle de ses ancêtres, l'orgueil de ses racines, racines qui sont les nôtres. Mais voici une copie de l'exemplaire du livre de Xocén [il le tient dans sa main et le montre à tous]! Nous félicitons la communauté pour avoir retrouvé son trésor. Je le garderai comme quelque chose de spécial, je le conserverai parmi les livres que je respecte le plus car dans ses pages réside la mémoire historique des Mexicains, celle qui nous rappelle d'où nous venons [tonnerre d'applaudissements] ${ }^{17}$.

Le discours laissait clairement entendre que les Mayas de Xocén avaient retrouvé leur livre sacré. Et c'est d'ailleurs en ces termes que le quotidien Diario del Sureste, organe de communication quasi officiel du PRI, relaya la nouvelle:

\section{[Le président] ajouta que son programme} de travail comprenait aussi la conservation des traditions et il se référa au livre de Xocén, perdu depuis plus de cent ans, qui avait été désormais retrouvé, et il rappela que le gouvernement fédéral avait soutenu les villageois dans leurs recherches ${ }^{\mathbf{1 8}}$.

La titraille allait encore plus loin : «Il emporte deux volumes du livre sacré de Xocén ». Seul le Diario de Yucatán, le journal conservateur de la bourgeoisie catholique, prit la peine de remarquer dans ses pages intérieures la «confusion» du président. Il rapportait ainsi qu'à la fin de l'assemblée, Fulgencio Noh avait pris une dernière fois la parole: il souhaitait préciser que le livre offert au président n'était en fait qu'une «compilation de traditions orales transmises par les anciens du village » et qu'il ne s'agissait donc pas du livre sacré ${ }^{19}$. Il n'était nulle part question d'Agueda Ruiz, restée dans les coulisses de l'événement.

La confusion du président déclencha une polémique de plusieurs jours dans la presse locale, qui accusa à plusieurs reprises «quelqu'un», une personne restée anonyme, d'avoir trompé le président en lui présentant la compilation comme une copie du livre perdu. L'erreur du président était-elle due à un malentendu ou à une manipulation intéressée? Question vaine de journalistes et de politiciens, qui s'affrontèrent, se lassèrent et passèrent à l'actualité suivante 20. Quant à Agueda Ruiz, dont on peut aisément imaǵiner le malaise face à l'inflation de la polémique, regrettant peut-être ses paroles à la fois naïves et ambiǵuës sur le «nouveau livre sacré de Xocén», elle se fit discrète dans les jours qui suivirent.

\section{$*$}

Ces nouvelles eurent un effet retentissant chez les habitants de Xocén. Tous les médias qui leur étaient accessibles affirmaient sans nuance, répétant les paroles du président, que le livre de Xocén avait été retrouvé. Plus encore: les photographies qui accompaǵnaient les articles montraient toutes le président avec le livre dans la main, promettant qu'il partirait avec et le conserverait soigneusement dans sa bibliothèque privée. La plupart des villageois n'avaient suivi que de très loin les activités des cinq membres du comité pour la recherche du livre sacré de Xocén. C'est donc par la presse qu'ils crurent découvrir à la fois que le livre avait été retrouvé et qu'il avait été remis au président. Ils apprirent également que Carlos Salinas de Gortari, le président des réformes néolibérales et du «consensus de Washinǵton», le président qui ambitionnait de diriger l'Organisation mondiale du commerce, le signataire de l'Alena, l'Accord de libre-échange nord-américain ratifié avec le Canada et les États-Unis et entré en vigueur au $1^{\mathrm{er}}$ janvier jour où l'Armée zapatiste de libération nationale déclencha son insurrection au Chiapas -, le président si souvent accusé par les journalistes d'avoir «vendu la patrie» aux intérêts étrangers ${ }^{21}$, ils apprirent donc que le président Carlos Salinas de Gortari était désormais reparti à México avec dans ses bagages le livre de Xocén. Ils pensèrent, atterrés, voir l'histoire se répéter.

Quand Fuǵencio Noh et Aǵueda Ruiz revinrent de Mérida, cette dernière fut immédiatement convoquée par une assemblée pour se justifier. Des explications furent données. Les villageois furent rassurés d'apprendre que le président n'avait emporté avec lui que la copie d'un rapport de recherche et qu'Agueda Ruiz n'avait fait que jouer avec les mots en prétendant reconstituer un équivalent du livre sacré. Cependant ils se rendirent compte que non seulement le livre n'avait pas été retrouvé après plus de deux années de recherche, mais que cette vaine quête avait aussi coûté beaucoup d'arǵent, arǵent dont la communauté n'avait pas profité pour d'autres projets peut-être plus prioritaires. La discussion dégénéra, d'autres problèmes, plus anecdotiques, refirent surface et Agueda Ruiz fut détenue pendant trois heures dans la prison du commissariat tandis que les autorités de Xocén rédigeaient son acte d'expulsion ${ }^{22}$. La recherche du livre des prophéties se terminait sur un échec total.

\section{$*$}

Aujourd'hui, le livre de Xocén est toujours considéré par les villageois comme disparu. La spoliation n'a en aucun cas été réparée. Les deux exemplaires du «nouveau livre sacré» confectionné par l'équipe d'Agueda Ruiz sont quant à eux conservés dans deux lieux hautement symboliques. L'un demeure la propriété de l'ex-président Carlos Salinas de Gortari, qui fut contraint à l'exil peu après la fin de son sexennat et qui reste jusqu'à présent l'un des présidents les
17. «Anuncian $\$ 32$ millones para créditos a la palabra»», Diario de Yucatán, 17 mai 1994; voir aussi: «Un millón de créditos a la palabra en el agro», Novedades de Yucatán, 17 mai 1994

18. «Lleva dos volumenes del Libro Sagrado de Xocén: Vivienda, producción y realidad étnica, tres etapas de la visita presidencial»», Diario del Sureste, 17 mai 1994

19. «Anuncian $\$ 32$ millones para créditos a la palabra», Diario de Yucatán, 17 mai 1994

20. «Siguen sin recuperar el famoso libro de Xocén», Diario de Yucatán, 18 mai 1994; «EI INI tiene copia de la obra que se dió al Presidente como si fuera el Libro de Xocén», Diario de Yucatán, 19 mai 1994; «Ese no es el Libro de Xocén - el Supremo Consejo Maya», Diario de Yucatán, 20 mai 1994; «Critican a quienes usan en beneficio propio a los mayas», Diario de Yucatán, 21 mai 1994.

21. Ayala Anguiano 1995, chap. XII; Favre et Lapointe (dir.) 1997: Salinas de Gortari 2000.

22. «Escándalo por el libro de Xocén: vecinos inconformes expulsan a una investigadora», Diario de Yucatán, 24 mai 1994; Mujica 1997: 10; Reed 2001: 355-356; Terán Contreras et Rasmussen 2005:91. «Expulsión del pueblo de la encargada de la búsqueda del Libro Sagrado», 19 mai 1994, in Terán Contreras et Rasmussen (éd.) 2007: 259-260. 
23. Terán Contreras et Rasmussen 2005: 72-73.

24. Voir aussi: Everton 2012 210, qui cite le témoignage d'Alfonso Dzib Nahuat.

25. Déléage 2017. plus impopulaires de l'histoire du Mexique. L'autre est conservé dans un coffre-fort de la bibliothèque de Xocén. Le premier exemplaire, expatrié, devenu inaccessible, peut-être même maintenant entre les mains des Occidentaux, a suivi le destin du livre de Xocén, confirmant l'acuité du discours géopolitique qui l'accompagnait. Le second, caché, invisible, est venu lui aussi rejoindre son modèle, peuplant l'imaǵinaire plutôt que le réel, horizon secret d'un mythe qui n'est toujours pas devenu réalité.

*

L'histoire du livre de Xocén apparaît comme une puissante réflexion concernant les relations conflictuelles que les Mayas entretiennent avec l'État mexicain et plus généralement avec les étrangers (qu'ils appellent les ts'uul). Cette réflexion, qui prend la forme d'une narration bien stabilisée et assez communément admise dans le village, comporte au moins deux dimensions qu'il importe de distinguer: la première est politique, la seconde épistémologique.

Il s'agiit d'abord du vol d'un livre, c'est-à-dire de l'anéantissement - plusieurs fois répété - d'un savoir traditionnel maya combiné à sa conservation secrète dans des archives et des mémoires éloignées, forcément étrangères. Ce récit de la dépossession d'un savoir, dont il est utile de rappeler qu'il était aussi livresque - les Mayas étant l'unique peuple amérindien à avoir inventé une écriture avant l'arrivée des conquistadors -, et de la perte corrélative d'autonomie politique était suffisamment évident pour avoir été compris et récupéré, malgré un emploi du temps chargé, par le président du Mexique.

La temporalité historique du récit est toutefois quelque peu nébuleuse. Le vol aurait pu être commis "au début du XIX siècle» selon une première version bien vite écartée, c'est-à-dire à l'époque de la colonie espaǵnole succédant à la conquête et aux autodafés des livres mayas. Il aurait pu avoir lieu «à la fin du XIX ${ }^{e}$ siècle », c'est-à-dire pendant la terrible guerre, connue sous le nom de "guerre des castes», qui opposa une partie des Mayas de la péninsule à l'État mexicain indépendant, lorsque l'église du village fut brûlée. Il aurait finalement pu prendre place au «milieu du $\mathrm{xx}^{\mathrm{e}}$ siècle », c'est-à-dire lors de l'intéǵration définitive de tous les Mayas de Xocén à l'économie de marché et plus largément au capitalisme de la région ${ }^{23}$. Autant de dates approximatives et sujettes à caution, qui rythment cependant une chronologie de la domination coloniale, républicaine et économique des Mayas. L'aspect spatial, géographique, du récit est beaucoup plus précis. On l'a vu, il dresse, par l'énumération hiérarchisée de toponymes - Chichimilá, Valladolid, Mérida, México, États-Unis -, une carte concentrique des degrés croissants de domination économique, technologique et politique auxquels est soumis le petit village maya.

L'histoire du livre de Xocén va toutefois plus loin que cette interprétation historique et géopolitique de la domination coloniale et néocoloniale. Elle laisse également transparaître une forme d'appréhension du savoir en général, une épistémologie. Car le contenu du livre des prophéties est en effet d'emblée relié à la technologie «américaine»: aux montres, aux ordinateurs, aux trains, aux voitures, aux avions, aux satellites et aux fusées. Ainsi, en 2007, un dignitaire religieux de Xocén pouvait parfaitement tenir des propos dans la continuité de ceux que nous avons déjà rapportés.

\section{Le livre de Xocén contient des prédictions} sur le monde et, étant donné son importance, il fut emporté à México et de là aux États-Unis, d'où il n'est jamais revenu. C'est parce que le livre est là-bas que les États-Unis ont une telle avance technologique, parce que toutes les inventions actuelles étaient décrites dans le livre.

(Licausi Pérez 2010: $142^{24}$ )

Le savoir scientifique est ainsi pensé dans ce récit maya non sur le mode de l'invention, mais sur celui de la révélation. Les Américains n'ont pas inventé la technologie moderne, ils l'ont découverte dans un livre. Et le savoir n'est pas le résultat d'une recherche scientifique ouverte, en perpétuelle mutation et en expansion continue, ni le fruit d'efforts intellectuels individuels et collectifs; il est toujours déjà là, donné, dans sa finitude et sa totalité, dans un unique livre. Les humains n'inventent donc pas un savoir nouveau: ils découvrent peu à peu, année après année, des parcelles d'un savoir qui existe déjà. Les Mayas de Xocén, au moins dans ce récit, pensent done l'invention comme une révélation, comme l'actualisation d'une prophétie.

Cette épistémologie de la révélation apparaît en continuité avec les diverses formes «d'anthropologies inversées » que j'ai étudiées dans une autre enquête, en Amazonie puis dans les Andes 25. Il s'aǵit chaque fois de penser l'origine des éléments les plus visibles d'une culture étrangère, les artefacts technologiques et plus généralement les marchandises, c'est-à-dire des objets dont les conditions de production (et non d'usage) sont éloignées à l'extrême des réalités quotidiennes et cognitives des Amérindiens. Ces raisonnements hypothétiques se stabilisent parfois et prennent alors la forme d'une oriǵine surnaturelle de ces marchandises - comme dans lesdits «cultes du cargo». Dans l'histoire du livre de Xocén, le savoir - et le pouvoir qu'il induit - est en effet d'oriǵine surnaturelle: non seulement il est issu d'un livre vivant, mais il prend la forme d'une prophétie, d'un savoir passé annonçant le futur.

\section{*}

Parmi les Mayas de la péninsule du Yucatán circulent aujourd'hui de très nombreuses prophéties. Elles se transmettent d'ordinaire oralement, même si certains pensent qu'elles proviennent d'un livre perdu (Pacheco Cruz 1960: 93 ; Sullivan 1983: 291; Vapnarsky 2017: 40). Ces prophéties fonctionnent 
parfois comme des éniǵmes : il faut alors deviner le sens caché d'un énoncé abscons. Souvent le sens ultime de ces prophéties a trait à une guerre à venir avec les étrangers ou à la fin prévisible d'un monde. Plus souvent encore il est à chercher dans les marchandises et la technologie modernes: dans les vêtements des étrangers, dans leurs routes, dans leurs véhicules - trains, voitures, autobus, avions, hélicoptères -, dans leurs moyens de communication - du télégraphe à la télévision selon les époques -, dans leur monnaie, etc. (Sullivan 1983 : 101-108; Sullivan 1989: 161-192; Lizama Quijano 2000; Terán Contreras et Rasmussen 2005 : 73-83 ; Vapnarsky 2017, chap. XI 26). En voici quelques exemples :

Le jour viendra où les fleurs d'une liane éclaireront ton chemin à la tombée de la nuit.

\section{La lumière électrique}

Le jour viendra où une personne te parlera de loin et où tu entendras tout ce qu'elle dira sans voir son visage.

\section{Le téléphone}

Le jour viendra où l'oiseau t'avalera avec tous tes enfants et, en arrivant à sa destination, les vomira tous.

\section{L'avion}

Le jour viendra où naîtra une énorme pierre qui ira de ville en ville, et son cheminement n'aura pas de fin, et par sa faute beaucoup de sang sera versé.

\section{La bombe atomique 27}

Prises ensemble, ces prophéties forment ce que Paul Sullivan a proposé de nommer une «théorie maya de l'histoire ${ }^{\mathbf{2 8}}$ ». Elles déploient une interprétation par fraǵments juxtaposés de l'avènement de la société moderne, depuis ses infrastructures de communication jusqu'à son économie capitaliste et guerrière. Et cette histoire se présente à chaque fois comme l'accomplissement d'une prophétie: le récit du passé semble ne pouvoir s'énoncer qu'au futur accompli. Selon Valentina Vapnarsky, les Mayas affirment ainsi, par la répétition de ces «prophéties sans prophète», que tout ce qui survient avait été prédit, que tout ce qui est nouveau avait déjà été dit: l'art de la prédiction devient chez eux art de la postdiction ${ }^{29}$. Ce qui n'est peut-être qu'une autre conséquence de l'épistémologie de la révélation et du savoir fini, donné une fois pour toutes et toujours déjà là, également au fondement de l'histoire du grand livre des prophéties de Xocén.

Aujourd'hui à Xocén, les villageois continuent eux aussi à se transmettre de semblables prophéties. Et ils insistent chaque fois sur le fait qu'elles proviennent du livre qui leur a été volé. Eux aussi savent d'où sont issus, en dernière instance, les ordinateurs, les avions,

\section{Le dernier livre}

Pierre Déléage

les satellites et les fusées. Et ils ajoutent à ce répertoire déjà très riche une ultime prophétie qui leur est propre.

Le livre reviendra, même si nous ne savons pas quand... Il va revenir dans son village. Et quand il reviendra, il y aura certainement de grands bouleversements dans la communauté, au niveau local, au niveau régional, et encore au-delà ${ }^{30}$.

Laboratoire d'anthropologie sociale pierre.deleage@college-de-france.fr
26. Natalio Noh et Fermín Dzib May le confirment également lors d'entretiens avec l'auteur à Xocén, les 26 novembre 2011 et 9 décembre 2011

27. Aké 1993: 39 (narrateurs: Alfonso Sotomaría et Primitivo Góngora)

28. Sullivan 1983: 13.

29. Vapnarsky 1999: chap. VII; Vapnarsky 2017: 405

30. Entretien de l'auteur avec Natalio Noh, Xocén, 26 novembre 2011.

Voir aussi: López Méndez 2003: 11. 


\section{Le dernier livre}

Pierre Déléage

\section{Bibliographie}

Aké, Santiago Domínguez

(éd.)

1993 Creencias, profecías $y$ consejas mayas. México, INI/Sedesol.

Ayala Anguiano, Armando 1995 Salinas y su México. México, Grijalbo.

\section{Boccara, Michel}

2017 Des os pour l'éternité philosophie et mythologie de la mort chez les Mayas du Yucatán. Paris, Maisonneuve et Larose/Hémisphères.

\section{Castellanos, María Bianet}

2010 A Return to Servitude: Maya Migration and the Tourist Trade in Cancún. Minneapolis, University of Minnesota Press.

\section{Déléage, Pierre}

2017 Lettres mortes. Paris, Fayard.

\section{Dzib May, Andrés}

1999 Breve reseña del pueblo de Xocén «El Centro del Mundo». Valladolid, Dirección General de Culturas Populares de Yucatán.

\section{Everton, Macduff,}

Demangeat, Charles et Hiler, Hilario

2012 The Modern Maya: Incidents of Travel and

Friendship in Yucatán.

Austin, University of Texas Press.

\section{Favre, Henr}

\section{et Lapointe,Marie (dir.)}

1997 Le Mexique, de la réforme néolibérale à la contre-révolution: la présidence de Carlos Salinas de Gortari, 1988-1994. Paris, L'Harmattan.

Gallart, María Antonieta et Rojas Rabiela, Teresa 2004 Arturo Warman. Biobibliografía. México, Universidad Nacional Autónoma de México.

\section{Licausi Pérez, Gabriela María}

2010 «Mitos, símbolos y territorio en Santa María de Chink'a' Dzono'ot y los designios del Fin del Mundo. Mémoire de maîtrise, Univers dad Autónoma de Yucatán.

Lizama Quijano, Jesús J.

2000 «La señales del fin del mundo: una aproximación a la tradición profética de los Cruzo'ob », in Genny Neǵroe Sierra et Francisco Fernández Repetto (dir.), Religión Popular De la reconstrucción histórica al análisis antropológico (aproximaciones casuísticas). Mérida, Universidad Autónoma de Yucatán: 133-162.
López Méndez, Roberto 2003 «Historia y leyenda de la Santa Cruz Tun de Xocén y el Sagrado Libro de la Vida», in Roberto López Méndez, Rocío López Arreguín et Luis Gutiérrez Yarce, Leyendas de vírgenes y santos de Yucatán. Mérida, Area Maya : 7-11.

Mujica, Sharon S.

1997 «Sacred Books. The Case of Xocen », communication personnelle de l'auteure, préparée pour le $\mathrm{xx}^{\mathrm{e}}$ Congrès international de la Latin American Studies Association (17-19 avril), Guadalajara, Mexique.

Muñoz Castillo, Fernando

2001 Teatro experimental en Yucatán (1949-1999) México, Escenología A. C.

Navarrete Maya, Laura

2000 «María Alicia Martínez Medrano », Diccionario de escritores mexicanos: siǵlo $X X$, t. V. México, Universidad Nacional Autónoma de México : 146-147.

Pacheco Cruz, Santiago

1960 (1947) Usos, costumbres, religión y supersticiones de los mayas. Mérida, Yucatán.

Reed, Nelson A.

2001 (1964) The Caste War of Yucatán. Revised Edition. Stanford, Stanford University Press.

Salinas de Gortari, Carlos

2000 México: un paso difícil a la modernidad. Barcelone, Plaza \& Janés.

\section{Sullivan, Paul Robert}

1983 Contemporary Yucatec Maya Apocalyptic Prophecy: The Ethnographic and Historical Context. Thèse de doctorat, Baltimore, Johns Hopkins University.

\section{Sullivan, Paul Robert}

1989 Unfinished Conversations. New York, Alfred A. Knopf.

Terán Contreras, Silvia et Rasmussen, Christian H. (dir.)

1992 Relatos del centro del mundo = U tsikbalo'obi chuumuk lu'um, t. I. Mérida, Gobierno del Estado de Yucatán.

Terán Contreras, Silvia et Rasmussen, Christian H. 2005 Xocén: el pueblo en el Centro de Mundo. Mérida, Universidad Autónoma de Yucatán.

Terán Contreras, Silvia et Rasmussen, Christian H. (éd.)

2007 Nohoch Libro de la comisaría de Xocén: documentos del archivo de la comisaría de 1930-1999. Mérida. Universidad Autónoma de Yucatán.

\section{Vapnarsky, Valentina}

1999 «Expressions et conceptions de la temporalité chez les Mayas Yucatèques du Quintana Roo (Mexique).» Thèse de doctorat, université Paris-Nanterre.

2017 «Les Sens du temps : temporalités et temporalisations des paroles, expériences et mémoires mayas.

Manuscrit soumis pour l'habilitation à diriger des recherches, École des hautes études en sciences sociales.

Warman, Arturo, Nolasco Armas, Margarita, Guillermo Bonfil, Olivera, Mercedes et Valencia, Enrique

1970 De eso que llaman antropologia mexicana. México, Nuestro Tiempo.

\section{Ci-contre et ouverture}

Le Président mexicain Carlos Salinas de Gortari lors de sa visite Xocén, 26 octobre 1990 Diario de Yucatán, Merida, Yucatán. Droits réservés. 
